\title{
Recombinant Human Bone Morphogenetic Protein-2 in Development and Progression of Oral Squamous Cell Carcinoma
}

\author{
Khaled Waleed Zaid ${ }^{1 *}$, Mansour Chantiri², Ghassan Bassit ${ }^{3}$
}

\begin{abstract}
Bone morphogenetic proteins (BMPs), belonging to the transforming growth factor- $\beta$ superfamily, regulate many cellular activities including cell migration, differentiation, adhesion, proliferation and apoptosis. Use of recombinant human bone morphogenic protein-2 (rhBMP-2) in oral and maxillofacial surgery has seen a tremendous increase. Due to its role in many cellular pathways, the influence of this protein on carcinogenesis in different organs has been intensively studied over the past decade. BMPs also have been detected to have a role in the development and progression of many tumors, particularly disease-specific bone metastasis. In oral squamous cell carcinoma - the tumor type accounting for more than $90 \%$ of head and neck malignancies- aberrations of both BMP expression and associated signaling pathways have a certain relation with the development and progression of the disease by regulating a range of biological functions in the altered cells. In the current review, we discuss the influence of BMPs -especially rhBMP-2- in the development and progression of oral squamous cell carcinoma.
\end{abstract}

Keywords: Oral squamous cell carcinoma - recombinant human bone morphogenetic protein-2 - carcinogenesis.

Asian Pac J Cancer Prev, 17 (3), 927-932

\section{Introduction}

Bone morphogenetic proteins (BMPs) compose a family of growth factors also known as cytokines and as metabologens (Reddi and Reddi, 2009), they also belong to the family of TGF- $\beta$ (Nana et al., 2015), which induces bone formation, regeneration and participates in many important steps during early embryonic development and homeostasis of diverse tissues and organs through regulating cellular differentiation(Kobayashi et al., 2005), proliferation (Chu et al., 2014; Peng et al., 2015), apoptosis (Peterkova et al., 1998; Kawamura et al., 2002; Hallahan et al., 2003) and motility (Perez et al., 2011; Kim et al., 2015).

BMPs play their role through a heteromeric receptor complex, which is composed of two types (type I and II) of serine-threonine kinase transmembrane receptors (Ghosh-Choudhury et al., 2002). Three subtype I receptors have been shown to bind BMP ligands, namely: subtype IA and IB and subtype IA-activin receptors (Granjeiro et al., 2005). Signal transduction studies have revealed that Smad1, 5 and 8 are the immediate downstream molecules of BMP receptors and play an essential role in BMP signal transduction (Chen et al., 2004). The physiologic roles of the BMPs include fundamental roles during embryonic maturation especially in the specification of positional information in the embryo and the skeletal and non-skeletal tissue arrangement (Hogan, 1996). Recently, Bone morphogenetic proteins is used as the osteoinductive elements in many tissue engineering products as replacements for autogenous bone graft, and for bone augmentation and repair.

BMP2 plays complicated roles, including the promotion of cell survival, regulation of cell proliferation, functional differentiation, cell motility, self-renewal and apoptosis of embryonic stem cells (Kang et al., 2009).

Recently many studies referred that BMPs play a pivotal role in tumor development (Sanders et al., 2014), progression and bone metastasis (Kwon et al., 2010; Secondini et al., 2011; Mock et al., 2015).

BMPs preserve the polarity of epithelial cells via intracellular signaling from BMP receptors. In polarized epithelial cells, BMP signals are transmitted from BMP receptor complexes exclusively localized at the basolateral surface of the cell membrane. Also, basolateral motivation with BMP increases the expression of components of tight junctions and increases the trans-epithelial resistance (TER) (Saitoh et al., 2013)

\section{Oral squamous cell carcinoma}

Oral cancer refers to cancer occurring between the

${ }^{l}$ Department of Oral Histology and Pathology, Faculty of Dentistry, Damascus University, ${ }^{3}$ Department of Oral Surgery and Implantology, IUST University , Damascus, Syrian Arab Republic, ${ }^{2}$ Department of Periodontology, Faculty of Dentistry, Lebanese University, Beirut, Lebanon*For correspondence:dr.khaled.zaid@gmail.com 
vermilion border of the lips and the junction of the hard and soft palates or the posterior one third of the tongue. As with most head and neck sites, squamous cell carcinoma is the most common oral cancer. This type of malignancies can arise in several places, but it is often preventable, and if diagnosed early is usually curable.

Oral squamous cell carcinoma (OSCC) composes About $95 \%$ of oral cancers in India (Krishna et al., 2014). The most important risk factors for oral SCC are: use of tobacco or betel quid and the regular drinking of alcoholic beverages (Zaid, 2014). However, infection with high-risk human papillomavirus (HPV) genotypes, and a diet low in fresh fruits and vegetables have also recently been implicated in the aetiopathogenesis of oral SCC (Petti, 2009) (Sasaki et al., 2015).

The attention to BMPs and their role in oral cancers has increased as considerable progress has been made in this area. The present review summarizes the current knowledge on the roles played by BMPs -especially rhBMP2- in oral squamous cell carcinoma.

\section{Bone morphogenetic protein-2 and Cancer}

The effects of BMP-2 on the malignant cells still controversial and are perhaps contingent upon the tissues and environment where they are expressed (Suzawa et al., 1999).

Many researchers investigated the link between BMPs and cancer. many of them have pointed that several types of BMPs (such as BMP-2, BMP-4,BMP-6 and BMP-7) are implicated in many types of cancer tissues and also many studies reported their Dysregulation. (Mancino et al., 2008; Le Page et al., 2009; Aoki et al., 2011; Hu et al., 2013; Kim et al., 2015).

In tumorigenesis, BMP2 signaling plays dual roles, functioning as cell context-dependent tumor suppressors or oncogenes, Therefore, perturbations in BMP2 signaling may lead to increased tumorigenesis. BMP2 ability to enhance tumorigenicity in vivo is mediated by stimulating angiogenesis, suppressing immune surveillance, or enhancing the degradation of ECM (Langenfeld and Langenfeld, 2004).

It has been reported: when rh-BMP-2 utilized clinically in spinal fusion surgery, it appears to promote carcinogenesis at higher rates than monitored in the overall population. Furthermore, BMP and TGF-beta are correlated with the increased cancer growth in both; the clinic and the laboratory.(Epstein, 2014).

The relationship between cancer cells and BMP-2 has been investigated in several studies. In a clinical research held by Carragee et al, spinal surgery with high-dose rhBMP-2 treatment led to four-fold increase in the number of newly formed malignancies after 24 months, compared to the untreated group, and the discrepancy increased after 60 months (Carragee et al., 2011).

Carragee et al also evaluated the influence of recombinant human bone morphogenetic protein-2 (rhBMP-2) in-vitro on the growth/invasiveness of cancer and have found that : A high dose of $40 \mathrm{mg}$ of rhBMP-2/ CRM in lumbar spinal arthrodesis was associated with an increased risk of new cancer. (Carragee et al., 2013)
BMP-2 reduces cellular proliferation of most cancer types such as colorectal, hepatocellular, osteosarcoma and renal cancer (Togo et al., 2008; Qiu et al., 2010; Wang et al., 2011; Wang et al., 2015). This protein has a certain roles in many tumors like lung cancer (Bieniasz et al., 2009; Fei et al., 2013) where The serum BMP-2 level is positively correlated with clinical stage and may serve as an independent negative predictor for prognosis (Fei et al., 2013), it also has no effects on prostate cancer growth or proliferation (Feeley et al., 2006).

Studies on oral, breast, gastric, colon, bladder and pancreatic cancer revealed that BMP-2 affects positively on the invasiveness of these cancers. (Clement et al., 2005; Kim et al., 2014; Kim et al., 2015).

In cancer cells, BMP-2 was found to repress apoptosis induced by $\mathrm{TNF} \alpha$ or by serum deprivation (Chen et al., 2001) and BMP-2 overexpression may modulate cellular motility and cellular adherence. The increased expression of BMP-2 in ovarian cancer tissues is associated with shorter survival in patients (Le Page et al., 2009).

\section{BMPs and biology of oral cancer cells}

Bone morphogenetic protein-2 (BMP-2) is the leading osteoinductive growth factor used clinically in bone-related regenerative medicine today, it has been used widely in the field of maxillofacial surgery and implantology.

(BMP-2)-containing bone grafts are recently considered as a useful regenerative materials for oral and maxillofacial surgery; however, several in vitro and in vivo studies previously indicated to the existence of cancer progression-related adverse effects caused by BMP-2 (Kim et al., 2014).

The biological role of BMP-2 in OSCC has not been elucidated but the roles of BMPs in the biology of head and neck cancer cells have been recently intensively investigated. It is now known that BMPs are extensively involved in the regulation of cellular functions of the cancer cells, ranging from cell growth and death, cell migration, invasion and epithelial to mesenchymal transition (EMT).

Qiao et al suggest that BMP-mediated EMT constitutes one possible pathway for the development of CSCs (cancer stem cells) in the OSCC, implying a transient therapeutic opportunity if EMT can be interrupted early in the development of the neoplasm. (Qiao et al., 2011)

\section{BMP-2 and fibroblasts in OSCC}

The influence of fibroblasts on oral cancer progression related to BMP-2 has not yet been considered, although stromal fibroblasts are known as essential and main host cell types in tumor microenvironments (Salo et al., 2014). Cancer-associated fibroblast cells are responsible for the manufacturing of paracrine growth factors, proteolytic enzymes, and extracellular matrix (ECM) components, by which the combination of cancer epithelial cells and fibroblasts promote tumorigenesis and development of tumors that are wider than malignant epithelia alone (Salo et al., 2014). 
Recombinant Human Bone Morphogenetic Protein-2 in Development and Progression of Oral Squamous Cell Carcinoma

Interestingly, many studies has indicated that stimulation of dermal fibroblasts by BMPs can enhance the secretion of pro-tumorigenic factors, and the treatment with BMP in turn elevates pro-tumorigenic secreted factors such as IL-6 and MMP-3. These findings indicate that BMP may stimulate tumor progression within the tumor microenvironment (Owens et al., 2013).

Fibroblasts act directly on the tumor cells to stimulate growth and evade apoptosis and also regulate the extracellular matrix or the microenvironment physical structure of the tumor by enzymatically modulating ExtraCellular Matrix (ECM) components such as , fibronectin, collagen and constituent of the basal lamina. Regulation of the stiffness and physical structure of the ECM can enhance the growth and metastatic dissemination of the tumor cells (Erler and Weaver, 2009). Fibroblasts can also regulate angiogenesis by stimulating a new vessel growth to support tumors progression (Yang et al., 2008).

BMPs have been considered to be global regulators of transcriptional networks, including the repression or silencing of genes (Koinuma et al., 2009). This family of growth factors, could have a unique function in fibroblasts came from a recent study demonstrating clear transcriptional reactions in human keratinocytes when compared to their underlying dermal fibroblasts. Intriguingly, a list of BMP motivated genes contained several factors that have been demonstrated to promote cancer progression, such as IL-11, CTGF, and ADAM12 (Fessing et al., 2010).

Inhibition of BMP signaling in fibroblasts may provide a successful adjuvant to current therapies, given that fibroblasts are usually released from mutations found within the tumor (Campbell et al., 2009).

In many tissues like the renal interstitial, BMP-2 is supposed to have the potential to attenuate TGF- $\beta 1$ induced fibrosis by attenuating Snail expression and reversing EMT process (Yang et al., 2011) but still no evidences or sufficient studies on the oral mucosa.

\section{BMP and cell cycle / proliferation.}

The balance between both differentiation and proliferation is essential for proper formation of various tissues and organs. If this balance is lost, then it would lead to the tumorigenesis. In normal cells such as muscle satellite cells descendants, BMP signaling plays a p role in balancing differentiation and proliferation of activated cells. Initially, BMP signals preserve satellite cells descendants in a proliferating state thereby expanding cell numbers. After cells are committed to differentiate they raise the expression of the BMP inhibitor Chordin, thereby supporting terminal differentiation and myotube formation in a negative feedback mechanism. (Friedrichs et al., 2011).

BMPs play important roles on Primordial germ cells (PGC) specification. BMP4 emanating from the extra embryonic ectoderm and BMP2 from the visceral endoderm induce the phosphorylation of SMAD1 and SMAD5, which form a complex with SMAD4. This complex moves into the nucleus and presumably binds enhancers and promoters of genes that are required to establish PGC fate. In addition, BMP signaling leads to direct or indirect activation of WNT3. (Gunesdogan et al., 2014) mostly, WNT3 signaling leads to suppression of GSK3, which in turn causes the stabilization of $\beta$-CATENIN. $\beta$-CATENIN moves into the nucleus and binds with LEF1/TCF leads to activating the transcription of various target genes including c-jun, c-myc, fra-1, cyclinD1 urokinase-type plasminogen activator receptor, then leading the tumor cells to proliferation, EMT, migration, and invasion. (Zaid, 2014) (Figure 1).

BMPs are able to regulate the growth of cancer cells. The cellular response is influenced by the individual BMP. Some BMPs show an inhibitory effect on proliferation of cancer cells, while others have a reverse effects.

BMP-2 inhibit the proliferation of cancer cells in the breast (Arnold et al., 1999). while, other BMPs may indirectly promote the proliferation of breast cancer cells, such as BMP-4 (Montesano et al., 2008).

BMPs can also co-regulate the growth of cancer cells induced by non-cytokine agents. For example, the up-regulation of $\mathrm{p} 21$ by BMP-2 can also prevent EGFinduced proliferation of cancer cells (MDA-MB-231) (Ghosh-Choudhury et al., 2000). BMP-2 has a direct anti-proliferative effect on tumor cells at a very high concentration $(1 \mu \mathrm{g} / \mathrm{ml})$ in vitro (Soda et al., 1998). Interesting ,the regulation of $\mathrm{p} 21$ expression by BMP-2 was mediated by Type-I receptors, Smad-1 and Smad-4. (Pouliot and Labrie, 2002).

\section{BMP-2 and the epithelial to mesenchymal transition}

Epithelial to mesenchymal transition (EMT) is a biological process required in the embryonic development for the formation of tissues which cells originate in locations far from their final destination. Cancer cells hijack this program for tumor dissemination (Ocana et al., 2012). EMT not only causes a disruption of epithelial
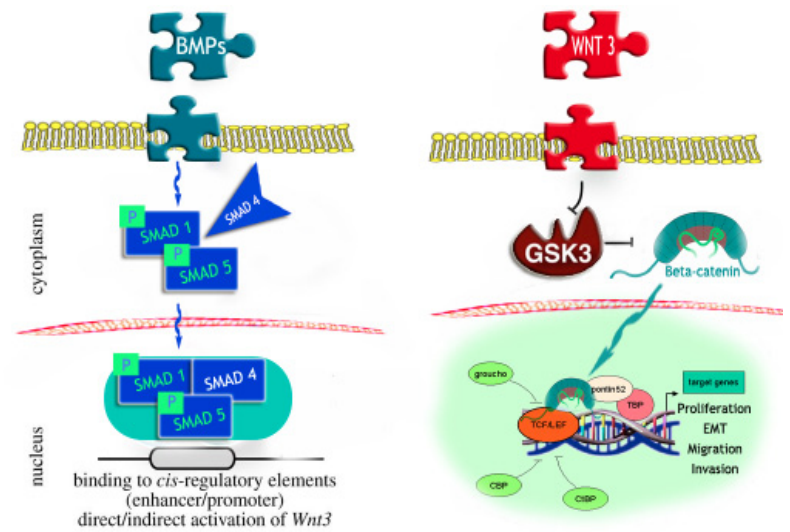

Figure 1. BMP2 Induces the Phosphorylation Process of SMAD5 and SMAD1, which form a Complex with SMAD4. This complex moves into the nucleus and binds promoters and enhancers of genes, leading to direct or indirect activation of WNT3, Most likely, WNT3 signaling causes the inhibition of GSK3, which causes the stabilization of $\beta$-CATENIN. $\beta$-CATENIN moves into the nucleus and together with LEF1/TCF lead to the cellular proliferation ,migration, invasion and epithelial to mesenchymal transition 


\section{Khaled Waleed Zaid et al}

homeostasis which may lead to carcinogenesis, it can also transform the indolent tumour cells into a more aggressive colony, leading to metastasis.

Studies showed that the bone morphogenetic proteins can enhance the reprogramming by activating EMT and the blockage of BMPs pathway would impair the reprogramming process (Samavarchi-Tehrani et al., 2010).

Kang et al indicated that prolonged BMP2 exposure of many cellular lines caused a destruction of E-cadherinmediated cell-cell contact and gain of a mesenchymal phenotype, which led to the increased cellular motility and invasiveness, also they found that obstruction of BMP2 signaling by BMP2 siRNA induced MET, indicating that BMP2 induces EMT and controls motility and invasiveness of cancer cells and plays an important role in promoting late-stage tumor progression (Kang et al., 2009).

Many recent studies suggested that BMP-2 induces EMT in gastric and pancreatic cancer cells, and this contributes to increased motility (Kang et al., 2010), However, the contribution of BMP-2 to EMT and its associated invasiveness in OSCC cells have not been yet characterized.

\section{BMP-2 and invasiveness of tumour cells}

Tissue invasion is of the six acquired capabilities of cancer cells as described by Hanahan and Weinberg (Hanahan and Weinberg, 2000). Kang et al, indicated that an increased concentration of BMP-2 strongly enhanced motility and invasiveness in gastric cancer cells, whereas no increase was noticed in cells treated with either Noggin (a BMP-2 inhibitor) or BMP-2 blocking antibodies. In vitro studies demonstrated that rhBMP-2 promotes the invasiveness of several human oral squamous cell carcinomas cell lines, and this effect is dependent, in part, on the baseline gene expression of BMP-2. This effect was observed for cell lines that demonstrated gene expression of BMP-2 (Kokorina et al., 2011).

Zhou et al. (2006) analyzed 25 tongue squamous cell carcinomas by gene microarrays and qPCR analyses and revealed that BMP-2 expression may be in relation with lymph node metastasis (Zhou et al., 2006).

Kim et al suggested that $1 \mathrm{ng} / \mathrm{ml}$ rhBMP- 2 may induce invasion of oral squamous cell carcinoma (OSCC) cells mediated by CCL5 release. Therefore, they advise to have a careful clinical examination before using rhBMP2-containing biomaterials to prevent cancer progression. Additionally, malignant tissues adjacent to the defective areas should be treated or removed in advance to eliminate the possibility of adverse effects caused by rhBMP-2 (Kim et al., 2014).

\section{rhBMP-2 and tumour related angiogenesis}

Angiogenesis is an essential event during the development and progression of both primary and secondary tumours. Langenfeld provided many evidences that BMP-2-induced angiogenesis occurs at least, in part, by stimulating endothelial cells. BMP-2 enhances tube formation, induces phosphorylation of Erk-1/2, Smad 1/5, and increases Id1 expression and that lead to activating endothelial cells (Langenfeld and Langenfeld, 2004). Erk-1/2 regulates several critical cellular functions in endothelial cells including proliferation and tube formation (Liu et al., 2001) ,Also many investigators indicated that Id has an essential role in mediating an angiogenic response during the embryonic development and on postnatal endothelial cells (Benezra, 2001).

The only available study on oral squamous cell carcinoma was held by Gao et al and they found that Exposing of OSCC cells to BMP-2 does not stimulate proliferation or angiogenesis (Gao et al., 2010).

rhBMP-2 and it's signalling play a profound role in the progression of Oral squamous cell carcinomas. Further investigation will elucidate the mechanisms underlying the involvement of BMPs in OSCC. It will expand current understanding for the pathogenesis and may provide clues for developing novel therapies in managing advanced diseases.

\section{References}

Aoki M, Ishigami S, Uenosono Y, et al (2011). Expression of BMP-7 in human gastric cancer and its clinical significance. Br J Cancer, 104, 714-8.

Arnold SF, Tims E, McGrath BE (1999). Identification of bone morphogenetic proteins and their receptors in human breast cancer cell lines: importance of BMP2. Cytokine, 11, 1031-7.

Benezra R (2001). Role of Id proteins in embryonic and tumor angiogenesis. Trends Cardiovasc Med, 11, 237-41.

Bieniasz M, Oszajca K, Eusebio M, et al (2009). The positive correlation between gene expression of the two angiogenic factors: VEGF and BMP-2 in lung cancer patients. Lung Cancer, 66, 319-26.

Campbell I, Polyak K, Haviv I (2009). Clonal mutations in the cancer-associated fibroblasts: the case against genetic coevolution. Cancer Res, 69, 6765-8.

Carragee EJ, Chu G, Rohatgi R, et al (2013). Cancer risk after use of recombinant bone morphogenetic protein-2 for spinal arthrodesis. J Bone Joint Surg Am, 95, 1537-45.

Carragee EJ, Hurwitz EL, Weiner BK (2011). A critical review of recombinant human bone morphogenetic protein-2 trials in spinal surgery: emerging safety concerns and lessons learned. Spine J, 11, 471-91.

Chen D, Zhao M, Mundy GR (2004). Bone morphogenetic proteins. Growth Factors, 22, 233-41.

Chen S, Guttridge DC, Tang E, et al (2001). Suppression of tumor necrosis factor-mediated apoptosis by nuclear factor kappaB-independent bone morphogenetic protein/Smad signaling. J Biol Chem, 276, 39259-63.

Chu H, Luo H, Wang H, et al (2014). Silencing BMP-2 expression inhibits A549 and H460 cell proliferation and migration. Diagn Pathol, 9, 123.

Clement JH, Raida M, Sanger J, et al (2005). Bone morphogenetic protein 2 (BMP-2) induces in vitro invasion and in vivo hormone independent growth of breast carcinoma cells. Int J Oncol, 27, 401-7.

Epstein NE (2014). Basic science and spine literature document bone morphogenetic protein increases cancer risk. Surg Neurol Int, 5, S552-60.

Erler JT, Weaver VM (2009). Three-dimensional context regulation of metastasis. Clin Exp Metastasis, 26, 35-49.

Feeley BT, Krenek L, Liu N, et al (2006). Overexpression of noggin inhibits BMP-mediated growth of osteolytic prostate cancer lesions. Bone, 38, 154-66.

Fei ZH, Yao CY, Yang XL, et al (2013). Serum BMP-2 up- 
Recombinant Human Bone Morphogenetic Protein-2 in Development and Progression of Oral Squamous Cell Carcinoma

regulation as an indicator of poor survival in advanced non-small cell lung cancer patients. Asian Pac J Cancer Prev, 14, 5293-9.

Fessing MY, Atoyan R, Shander B, et al (2010). BMP signaling induces cell-type-specific changes in gene expression programs of human keratinocytes and fibroblasts. $J$ Invest Dermatol, 130, 398-404.

Friedrichs M, Wirsdoerfer F, Flohe SB, et al (2011). BMP signaling balances proliferation and differentiation of muscle satellite cell descendants. BMC Cell Biol, 12, 26.

Gao Q, Tong W, Luria JS, et al (2010). Effects of bone morphogenetic protein-2 on proliferation and angiogenesis in oral squamous cell carcinoma. Int J Oral Maxillofac Surg, 39, 266-71.

Ghosh-Choudhury N, Abboud SL, Nishimura R, et al (2002). Requirement of BMP-2-induced phosphatidylinositol 3-kinase and Akt serine/threonine kinase in osteoblast differentiation and Smad-dependent BMP-2 gene transcription. J Biol Chem, 277, 33361-8.

Ghosh-Choudhury N, Woodruff K, Qi W, et al (2000). Bone morphogenetic protein-2 blocks MDAMB 231 human breast cancer cell proliferation by inhibiting cyclin-dependent kinase-mediated retinoblastoma protein phosphorylation. Biochem Biophys Res Commun, 272, 705-11.

Granjeiro JM, Oliveira RC, Bustos-Valenzuela JC, et al (2005). Bone morphogenetic proteins: from structure to clinical use. Braz J Med Biol Res, 38, 1463-73.

Gunesdogan U, Magnusdottir E, Surani MA (2014). Primordial germ cell specification: a context-dependent cellular differentiation event [corrected]. Philos Trans $R$ Soc Lond B Biol Sci,369.

Hallahan AR, Pritchard JI, Chandraratna RA, et al (2003). BMP2 mediates retinoid-induced apoptosis in medulloblastoma cells through a paracrine effect. Nat Med, 9, 1033-8.

Hanahan D, Weinberg RA (2000). The hallmarks of cancer. Cell, 100, 57-70.

Hogan BL (1996). Bone morphogenetic proteins: multifunctional regulators of vertebrate development. Genes Dev, 10, 1580-94.

Hu F, Meng X, Tong Q, et al (2013). BMP-6 inhibits cell proliferation by targeting microRNA-192 in breast cancer. Biochim Biophys Acta, 1832, 2379-90.

Kang MH, Kang HN, Kim JL, et al (2009). Inhibition of PI3 kinase/Akt pathway is required for BMP2-induced EMT and invasion. Oncol Rep, 22, 525-34.

Kang MH, Kim JS, Seo JE, et al (2010). BMP2 accelerates the motility and invasiveness of gastric cancer cells via activation of the phosphatidylinositol 3-kinase (PI3K)/Akt pathway. Exp Cell Res, 316, 24-37.

Kawamura C, Kizaki M, Ikeda Y (2002). Bone morphogenetic protein (BMP)-2 induces apoptosis in human myeloma cells. Leuk Lymphoma, 43, 635-9.

Kim BR, Oh SC, Lee DH, et al (2015). BMP-2 induces motility and invasiveness by promoting colon cancer stemness through STAT3 activation. Tumour Biol.

Kim MJ, Kim KM, Kim J, et al (2014). BMP-2 promotes oral squamous carcinoma cell invasion by inducing CCL5 release. PLoS One, 9, 108170.

Kobayashi T, Lyons KM, McMahon AP, et al (2005). BMP signaling stimulates cellular differentiation at multiple steps during cartilage development. Proc Natl Acad Sci U S A, 102, 18023-7.

Koinuma D, Tsutsumi S, Kamimura N, et al (2009). Promoterwide analysis of Smad4 binding sites in human epithelial cells. Cancer Sci, 100, 2133-42.

Kokorina NA, Zakharkin SO, Krebsbach PH, et al (2011). Treatment effects of rhBMP-2 on invasiveness of oral carcinoma cell lines. Laryngoscope, 121, 1876-80.

Krishna A, Singh RK, Singh S, et al (2014). Demographic risk factors, affected anatomical sites and clinicopathological profile for oral squamous cell carcinoma in a north Indian population. Asian Pac J Cancer Prev, 15, 6755-60.

Kwon H, Kim HJ, Rice WL, et al (2010). Development of an in vitro model to study the impact of BMP-2 on metastasis to bone. J Tissue Eng Regen Med, 4, 590-9.

Langenfeld EM, Langenfeld J (2004). Bone morphogenetic protein-2 stimulates angiogenesis in developing tumors. Mol Cancer Res, 2, 141-9.

Le Page C, Puiffe ML, Meunier L, et al (2009). BMP-2 signaling in ovarian cancer and its association with poor prognosis. J Ovarian Res, $2,4$.

Liu S, Yu D, Xu ZP, et al (2001). Angiogenin activates Erk1/2 in human umbilical vein endothelial cells. Biochem Biophys Res Commun, 287, 305-10.

Mancino M, Strizzi L, Wechselberger C, et al (2008). Regulation of human Cripto- 1 gene expression by TGF-beta 1 and BMP4 in embryonal and colon cancer cells. J Cell Physiol, 215, 192-203.

Mock K, Preca BT, Brummer T, et al (2015). The EMT-activator ZEB1 induces bone metastasis associated genes including BMP-inhibitors. Oncotarget, 6, 14399-412.

Montesano R, Sarkozi R, Schramek H (2008). Bone morphogenetic protein- 4 strongly potentiates growth factorinduced proliferation of mammary epithelial cells. Biochem Biophys Res Commun, 374, 164-8.

Nana AW, Yang PM, Lin HY (2015). Overview of Transforming Growth Factor beta Superfamily Involvement in Glioblastoma Initiation and Progression. Asian Pac J Cancer Prev, 16, 6813-23.

Ocana OH, Corcoles R, Fabra A, et al (2012). Metastatic colonization requires the repression of the epithelialmesenchymal transition inducer Prrx1. Cancer Cell, 22, 709-24.

Owens P, Polikowsky H, Pickup MW, et al (2013). Bone Morphogenetic Proteins stimulate mammary fibroblasts to promote mammary carcinoma cell invasion. PLoS One, $\mathbf{8}$, e67533.

Peng J, Yoshioka Y, Mandai M, et al (2015). The BMP signaling pathway leads to enhanced proliferation in serous ovarian cancer-a potential therapeutic target. Mol Carcinog.

Perez VA, Ali Z, Alastalo TP, et al (2011). BMP promotes motility and represses growth of smooth muscle cells by activation of tandem Wnt pathways. J Cell Biol, 192, 171-88.

Peterkova R, Peterka M, Vonesch JL, et al (1998). Correlation between apoptosis distribution and BMP-2 and BMP-4 expression in vestigial tooth primordia in mice. Eur J Oral Sci, 106, 667-70.

Petti S (2009). Lifestyle risk factors for oral cancer. Oral Oncol, 45, 340-50.

Pouliot F, Labrie C (2002). Role of Smad 1 and Smad4 proteins in the induction of p21WAF1,Cip1 during bone morphogenetic protein-induced growth arrest in human breast cancer cells. $J$ Endocrinol, 172, 187-98.

Qiao B, Johnson NW, Chen X, et al (2011). Disclosure of a stem cell phenotype in an oral squamous cell carcinoma cell line induced by BMP-4 via an epithelial-mesenchymal transition. Oncol Rep, 26, 455-61.

Qiu H, Yang B, Pei ZC, et al (2010). WSS25 inhibits growth of xenografted hepatocellular cancer cells in nude mice by disrupting angiogenesis via blocking bone morphogenetic protein (BMP)/Smad/Id1 signaling. J Biol Chem, 285, 32638-46.

Reddi AH, Reddi A (2009). Bone morphogenetic proteins (BMPs): from morphogens to metabologens. Cytokine 
Khaled Waleed Zaid et al

Growth Factor Rev, 20, 341-2.

Saitoh M, Shirakihara T, Fukasawa A, et al (2013). Basolateral BMP signaling in polarized epithelial cells. PLoS One, $\mathbf{8}$, 62659.

Salo T, Vered M, Bello IO, et al (2014). Insights into the role of components of the tumor microenvironment in oral carcinoma call for new therapeutic approaches. Exp Cell Res, 325, 58-64.

Samavarchi-Tehrani P, Golipour A, David L, et al (2010). Functional genomics reveals a BMP-driven mesenchymalto-epithelial transition in the initiation of somatic cell reprogramming. Cell Stem Cell, 7, 64-77.

Sanders AJ, Ye L, Li J, et al (2014). Tumour angiogenesis and repulsive guidance molecule b: a role in HGF- and BMP-7mediated angiogenesis. Int J Oncol, 45, 1304-12.

Sasaki T, Kishimoto S, Kawabata K, et al (2015). Risk factors for cervical lymph node metastasis in superficial head and neck squamous cell carcinoma. J Med Dent Sci, 62, 19-24.

Secondini C, Wetterwald A, Schwaninger R, et al (2011). The role of the BMP signaling antagonist noggin in the development of prostate cancer osteolytic bone metastasis. PLoS One, 6, 16078.

Soda H, Raymond E, Sharma S, et al (1998). Antiproliferative effects of recombinant human bone morphogenetic protein-2 on human tumor colony-forming units. Anticancer Drugs, 9, 327-31.

Suzawa M, Takeuchi Y, Fukumoto S, et al (1999). Extracellular matrix-associated bone morphogenetic proteins are essential for differentiation of murine osteoblastic cells in vitro. Endocrinol, 140, 2125-33.

Togo N, Ohwada S, Sakurai S, et al (2008). Prognostic significance of BMP and activin membrane-bound inhibitor in colorectal cancer. World J Gastroenterol, 14, 4880-8.

Wang L, Park P, La Marca F, et al (2015). BMP-2 inhibits tumorinitiating ability in human renal cancer stem cells and induces bone formation. J Cancer Res Clin Oncol, 141, 1013-24.

Wang L, Park P, Zhang H, et al (2011). BMP-2 inhibits the tumorigenicity of cancer stem cells in human osteosarcoma OS99-1 cell line. Cancer Biol Ther, 11, 457-63.

Yang S, Pham LK, Liao CP, et al (2008). A novel bone morphogenetic protein signaling in heterotypic cell interactions in prostate cancer. Cancer Res, 68, 198-205.

Yang YL, Ju HZ, Liu SF, et al (2011). BMP-2 suppresses renal interstitial fibrosis by regulating epithelial-mesenchymal transition. J Cell Biochem, 112, 2558-65.

Zaid KW (2014). Immunohistochemical Assessment of E-cadherin and beta-catenin in the Histological Differentiations of Oral Squamous Cell Carcinoma. Asian Pac J Cancer Prev, 15, 8847-53.

Zhou X, Temam S, Oh M, et al (2006). Global expression-based classification of lymph node metastasis and extracapsular spread of oral tongue squamous cell carcinoma. Neoplasia, 8, 925-32. 